\title{
Prevalencia de infecciones nosocomiales en niños: encuesta de 21 hospitales en México
}

\author{
Carlos Avila-Figueroa, M.C., ${ }^{(1)}$ Miguel C ashat-C ruz, M.C., ${ }^{(1)}$ Eduardo Aranda-Patrón, M.C., ${ }^{(2)}$ \\ Angel R. León, M.C., ${ }^{(3)}$ N ancy Justiniani, M.C., ${ }^{(4)}$ Lucía Pérez-Ricárdez, M.C., ${ }^{(5)}$ Francisco Avila-C ortés, M.C. .( ${ }^{(6)}$ \\ Manuel Castelán, M.C., ${ }^{(7)}$ Roberto Becerril, M.C., ${ }^{(8)}$ Enedina Luz Herrera, M.C. ${ }^{(9)}$
}

\author{
Avila-Figueroa C, Cashat-Cruz M, Aranda-Patrón E, \\ León AR, Justiniani N, Pérez-Ricárdez $L$, \\ Avila-Cortés F, Castelán M, Becerril R, Herrera EL. \\ Prevalencia de infecciones nosocomiales \\ en niños: encuesta de 21 hospitales en México. \\ Salud Publica Mex 1999;41 suppl 1:S18-S25.
}

\section{Resumen}

Objetivo. D eterminar la prevalencia de infecciones nosocomiales, los factores de riesgo, su microbiología, la prevalencia de uso de antibióticos y la mortalidad asociada en niños hospitalizados. Material y métodos. Se realizó un estudio transversal en 21 hospitales públicos que atienden a niños. Se incluyeron pacientes desde recién nacidos hasta de 17 años de edad. Los riesgos ajustados se estimaron mediante regresión logística múltiple. Resultados En 1183 niños estudiados se identificaron 116 pacientes con infección nosocomial, con una prevalencia de $9.8 \%$ (IC 95\% 8.1-11.6). LoS sitios de infección más frecuentes fueron: neumonía (25\%), sepsis/bacteriemia (19\%) e infección del tracto urinario (5\%). El principal microrganismo identificado en hemocultivo fue Klebsiella pneumoniae (31\%). La prevalencia de uso de antibióticos fue de $49 \%$ con una variación de entre 3 y $83 \%$. Mediante regresión logística múltiple se encontraron cuatro factores independientemente asociados con el desarrollo de infección nosocomial: exposición a catéteres intravenosos (RM 3.3, IC 95\% 1.9-5.9), alimentación parenteral (RM 2.1 , IC 95\% 1.0-4.5), ventilación mecánica (RM 2.3, IC 95\% 1.2-4.1) y ser recién nacido de bajo peso (RM 2.6, IC 95\% 1.0-6.8). La mortalidad general fue de 4.8\%; sin embargo, el riesgo de morir en pacientes con infección nosocomial fue del doble comparado con los no infectados (RM 2.6, IC $95 \%$ 1.3-5.1). Conclusiones Esta evaluación rápida, usando una metodología estándar, ha permitido caracterizar la epidemiología de las infecciones nosocomiales en niños. Los re-

\author{
Avila-Figueroa C, Cashat-Cruz M, Aranda-Patrón E, \\ León AR, Justiniani N, Pérez-Ricárdez $L$, \\ Avila-Cortés F, Castelán M, Becerril R, Herrera EL. \\ Nosocomial infections in children: \\ Prevalence survey in 21 public hospitals in Mexico. \\ Salud Publica Mex 1999;41 suppl 1:S18-S25.
}

\begin{abstract}
A bstract
Objective. The purpose of this study was to determine the prevalence of nosocomial infections, asso ciated risk factors, microbiology, use of antibiotics, and associated mortality among hospitalized children. Material and methods. A 1-day prevalence survey was conducted among 1183 children hospitalized in a nationwide network of 21 public hospitals caring for children. To attain consistency between hospitals, CDC nosocomial infection definitions were used. Adjusted relative odds of bacteremia were estimated using logistic regression analysis. Results. The prevalence of noso comial acquired infection was $9.8 \%$ (Cl 95\%, 8.1-11.6). The more prevalent infections were pneumonia $(25 \%)$, sepsis/bacteremia (19\%), and urinary tract infection (5\%). The main microorganism isolated in blood cultures drown from patients with nosocomial infection was K. penumoniae (31\%). The prevalence of antibiotics use was $49 \%$ with substantial variation between hospitals (range 3-83\%). U sing lo gistic regression analysis, four factors were independently associated with the risk of nosocomial infection: central venous catheters (OR 3.3; $\mathrm{CI} 95 \%$ 1.0-5.9), total parenteral nutrition (OR 2.1; CI 95\%1.0-4.5) mechanical ventilation (OR 2.3; $\mathrm{Cl} 95 \%$ 1.2-4.1), and low birth weight (O R 2.6; CI 95\% 1.0-6.8). The overall mortality was 4.8\%; however, patients with nosocomial infection had two times greater risk to die as compared to non-infected children (OR 2.6; CI 95\% 1.35.1). Conclusions. This rapid assessment survey using a standard methodology allows to document the prevalence
\end{abstract}

(1) Hospital Infantil de México Federico Gómez, México.

(2) Hospital General Dr. Manuel Gea González, México.

(3) Hospital Infantil de Sinaloa, México.

(4) Hospital General de México, México

(5) Hospital del N iño Poblano, Puebla, México,

(6) Hospital Infantil de Morelia Eva Sámano de López Mateos, Michoacán, México.

(7) Hospital General de Veracruz, México.

(8) Hospital del Niño DIFEM Toluca, Estado de México, México.

(9) Dirección General de Servicios de Salud del Distrito Federal, México.

Fecha de recibido: 19 de febrero de 1998 - Fecha de aprobado: 6 de agosto de 1998 Solicitud de sobretiros: D r. Carlos Avila. Hospital Infantil de México Federico Gómez, Departamento de Epidemiología, D octor Márquez N 0. 162, colonia D octores, 06720 México, D.F. 
sultados dieron lugar a programas preventivos dirigidos específicamente al cuidado de catéteres intravasculares y al uso de ventilación asistida, a fin de reducir la ocurrencia de sepsis/bacteriemia y neumonías, infecciones nosocomiales de alta prevalencia y mortalidad.

Palabras clave: infección hospitalaria; hospitales pediátricos; México of nosocomial infections in children. The results were used to develop targeted programs on central catheters and mechanical ventilation aimed to reduce bacteremia/sepsis and pneumonia, two nosocomial infections characterized by high prevalence and mortality.

Key words: nosocomial infection; hospitals, pediatric; Mexico
$\mathrm{L}$ as infecciones nosocomiales (IN) son importantes porque producen daños a la salud, aumentan los días de estancia hospitalaria de los pacientes, así como el uso de recursos de diagnóstico y tratamiento, y, sobre todo, porque todos estos efectos son potencialmente prevenibles. El riesgo de enfermar e incluso de morir por una infección que no era el motivo de ingreso al hospital está estrechamente vinculado a la calidad de la atención en los hospitales. Es por ello que se requieren programas de vigilancia encaminados a prevenir y controlar las IN.

El estudio sobre la eficacia para el control de IN en Estados Unidos de América (EUA) mostró que, mediante sistemas de vigilancia y programas de control, ${ }^{1}$ era posible prevenir hasta un tercio de este tipo de infecciones.

Para que los programas de control resulten exitosos, es necesario conocer la epidemiología de estas infecciones, su prevalencia, los microrganismos causales y los factores de riesgo asociados. Así por ejemplo, se ha observado que el conocimiento de las tasas de infección es un elemento muy importante para que las políticas de control sean efectivas. ${ }^{2,3}$

En México existe información aislada sobre IN en pediatría; la mayoría de los estudios están limitados a algunos hospitales de tercer nivel, y muy pocos abarcan servicios de pediatría en hospitales generales. Se han notificado tasas de infección por cada 100 egresos de 8.8 y 10 en el Hospital Infantil de México, ${ }^{4,5}$ de 9.7 en el Instituto Nacional de Pediatría ${ }^{6}$ y de 9.1 en el Hospital Pediátrico del Instituto Mexicano del Seguro Social (IMSS). ${ }^{7}$ En esos hospitales las tasas más altas de IN han correspondido a los recién nacidos. ${ }^{8}$ Las unidades de pediatría en hospitales de segundo nivel han notificado tasas de IN de 31.1 entre niños internados en el Hospital Gea González, ${ }^{9}$ y de 7.6 en el Hospital de Río Blanco en Veracruz. ${ }^{10}$ Esas tasas contrastan con las informadas en EUA de 4.1 por 100 egresos en hospitales pediátricos y de 1.2 en unidades de pediatría de hospitales generales. ${ }^{11}$ Sin embargo, toda esta información publicada es difícil de generalizar en pediatría, lo que motivó que varios hospitales mexicanos que atienden niños constituyeran el Grupo Interinstitucional para el Control de las Infecciones Nosocomiales en Pediatría. Su objetivo es desarrollar un programa interinstitucional para el control de estas infecciones en niños y representa el primer esfuerzo para organizar una red pediátrica que más tarde se integre a la Red Hospitalaria de Vigilancia Epidemiológica (RHO$\mathrm{VE})$, que en la actualidad desarrolla el sector salud en México. ${ }^{12}$

Este estudio se realizó con el objetivo de elaborar un diagnóstico de la situación de las IN en pediatría a fin de obtener, en forma confiable, rápida y económica, información sobre: a) la tasa de prevalencia de IN; b) los factores de riesgo asociados; c) los principales microrganismos involucrados; d) los patrones de uso de antibióticos, y e) la mortalidad asociada.

\section{Material y métodos}

Entre 1996 y 1997 se llevó a cabo un estudio transversal para determinar la prevalencia puntual de IN pediátricas. Se aplicó una encuesta a todos los pacientes pediátricos que ingresaron al hospital el día de la encuesta; ésta incluyó desde recién nacidos hasta adolescentes de 17 años de edad, y cuya estancia en el hospital rebasara las 48 horas.

\section{Hospitales participantes}

El estudio comprendió 21 hospitales públicos que atienden niños (figura 1), de los cuales nueve son hospitales pediátricos de referencia, con más de cinco subespecialidades, que atienden a pacientes de tercer nivel: Hospital Infantil de Tamaulipas, Hospital para el Niño Poblano, Hospital Infantil de Morelia, Hospital del Niño de Hidalgo, Hospital Infantil de Sonora, Hospital del Niño de Tabasco, Hospital Infantil de Sinaloa, Hospital del Niño de Toluca, Hospital Infantil de México Federico Gómez. Nueve son hospitales pediátricos de segundo nivel de atención: Hospital Pediátrico de Tacubaya, Hospital Pediátrico de Legaria, Hospital Pediátrico de Coyoacán, Hospital Pediátrico 


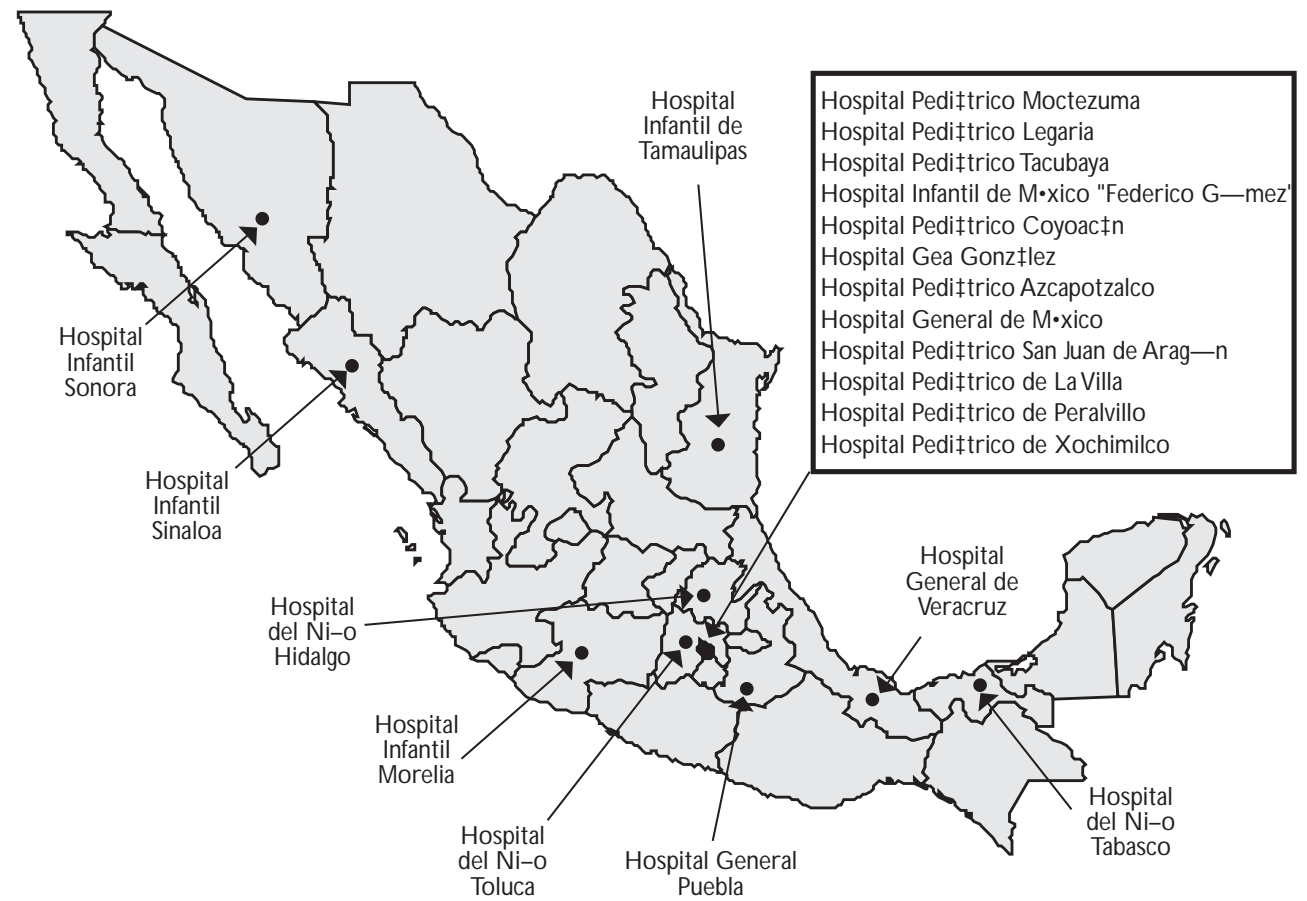
Figura 1. Hospitales participantes en la encuesta de Prevalencia de Infecciones Nosocomiales en Pediatría.
méxico, 1996-1997

de La Villa, Hospital Pediátrico Moctezuma, Hospital Pediátrico de Azcapotzalco, Hospital Pediátrico de San Juan de Aragón, Hospital Pediátrico de Peralvillo, Hospital Pediátrico de Xochimilco. Por último, tres son unidades de pediatría en hospitales generales: Hospital Manuel Gea-González, Hospital General de México, y Hospital General de Veracruz.

\section{Recolección de datos}

Después de invitar a los hospitales a participar, se distribuyó el protocolo, que fue aprobado por los comités de investigación de los nosocomios participantes. Se efectuaron varias reuniones en las que se brindó adiestramiento para el llenado de un formato precodificado acompañado de su instructivo. Este formato único se aplicó en todos los hospitales, y lo llenó el epidemiólogo participante en cada hospital, auxiliado por personal de enfermería. El levantamiento de las encuestas en cada hospital estaba planeado para un solo día, aunque dependiendo del tamaño del hospital y de los recursos, éste se realizó en el transcurso de uno a cinco días. Los datos se recolectaron en los diferentes hospitales entre los meses de marzo y junio de 1996. El formato se llenó junto a la cama del paciente, inves- tigando los siguientes aspectos: información demográfica (edad y sexo), diagnóstico de base, cirugía, procedimientos invasivos (catéteres intravasculares, urinarios, intubación endotraqueal, ventilación mecánica), prescripción de antibióticos, severidad de la enfermedad, así como la presencia de IN el día de la encuesta. Esta información se recogió de fuentes directas como el expediente clínico, las hojas de enfermería, exámenes de laboratorio, estudios de gabinete relevantes e, incluso, discusión con el médico o residente tratante. Para conocer la evolución final del paciente, se recogió información sobre sus condiciones al egreso, así como los resultados de bacteriología.

\section{Definiciones de IN}

Se consideró que una IN era aquella documentada clínicamente después de 48 horas de estancia en el hospital y que no estaba presente al ingreso. La presencia de una IN, de acuerdo con los diferentes sitios o localizaciones, se documentó con base en las definiciones operacionales que se utilizan en el Hospital Infantil de México y en el Hospital Infantil de Sonora, y que se distribuyeron entre los representantes de los hospitales participantes. Estas definiciones son estándar, 
pues se han publicado previamente ${ }^{4} \mathrm{y}$ se han adaptado a partir de las que han publicado los Centros para la Prevención y el Control de Enfermedades (CDC) de EUA, ${ }^{13} y$ de textos acerca de IN en niños. ${ }^{14}$

\section{A nálisis estadístico}

El análisis de los datos fue descriptivo y se presenta como distribución de frecuencias. También se estimaron intervalos de confianza (IC) al 95\%, de los estimadores de prevalencia y de riesgo (razón de momios -RM-) mediante métodos convencionales. Los riesgos se determinaron comparando las exposiciones previas al inicio de la infección en los infectados, con exposiciones el día de la encuesta en los no infectados. Para controlar el efecto de variables confusoras e identificar el efecto independiente de los factores de riesgo investigados, se efectuó análisis multivariado mediante regresión logística ${ }^{15}$ utilizando el paquete estadístico SAS. La mortalidad atribuible se calculó con métodos convencionales. ${ }^{16}$

\section{Resultados}

\section{Características generales de la población estudiada}

El análisis se llevó a cabo con 1039 niños hospitalizados; $85 \%$ estaban en alguno de los 18 hospitales pediátricos y $15 \%$ eran atendidos en alguna de las salas de pediatría de los tres hospitales generales participantes. Estos hospitales, en conjunto, brindan atención anualmente a 68000 niños. La estancia hospitalaria promedio fue de 16 días. Se incluyeron 533 hombres y 506 mujeres con una edad promedio de 4 meses. Los hospitales se clasificaron en tres grupos de acuerdo con el tipo de atención que proporcionan: a) servicios de pediatría en hospitales generales; $b$ ) hospitales pediátricos de segundo nivel de atención, y c) hospitales pediátricos de referencia que brindan atención de tercer nivel y que cuentan con más de cinco subespecialidades.

\section{Procedimientos invasivos}

Se encontró información relacionada con procedimientos invasivos, tanto diagnósticos como terapéuticos, de 1062 pacientes (89.8\%). El día del estudio había 583 pacientes que recibían soluciones intravenosas (56\%); 177 tenían un catéter intravascular largo (15\%); 64 recibían alimentación parenteral (5.4\%); 120 estaban con ventilación mecánica asistida (11.5\%), y 95 tenían colocado un catéter urinario (8\%).

\section{Utilización de antibióticos}

Hubo un total de 579 niños que recibían antibióticos el día del estudio (49\%). Estas prescripciones variaron de un hospital a otro, entre 3 y $83 \%$. Del total de prescripciones, $74 \%$ se catalogaron como terapéuticas, y $26 \%$, como profilácticas. De los niños con estas prescripciones, 39.7\% estaban recibiendo monoterapia, y $60.3 \%$, alguna combinación de antibióticos. La monoterapia y la combinación de antibióticos que con más frecuencia se administraron fueron la penicilina y la ampicilina-amikacina en 16.2 y $22 \%$ de los pacientes, respectivamente. Una cefalosporina de tercera generación, sola o en combinación, se administraba a 5.1\% de los pacientes.

\section{Prevalencia de infección nosocomial}

De los 1183 niños estudiados, se identificaron 116 con IN, con una prevalencia de 9.8\% (IC95\% 8.1-11.6). La prevalencia de IN varió entre los hospitales desde cero hasta 29 pacientes infectados por cada cien hospitalizados. De los niños, 2\% tenían más de una IN. De los 116 pacientes con IN, 32\% habían estado hospitalizados al día de la encuesta desde hacía más de una semana, y $18 \%$ tenían más de dos semanas en el hospital. La prevalencia de IN según el tipo de hospital se presenta en el cuadro I. Se observa un claro aumento en la prevalencia de infecciones en hospitales de mayor complejidad.

La prevalencia más alta de IN según el grupo de edad, fue de $13 \%$ en niños menores de un año $(p<0.01)$; no hubo diferencias en otros grupos. La prevalencia de infección en niños de entre 1 y 5 años fue de $9.4 \%$; en niños de entre 5 y 9 años, de $7.2 \%$, y en los mayores de 10 años, de $6.9 \%$. El promedio de estancia hospitalaria fue de 15.1 días en los niños con infección y de 10.4 días en los no infectados $(p<0.05)$.

\section{Sitios de IN}

Las localizaciones de infección más comunes fueron: neumonía (25\%), sepsis (19\%), bacteriemia (5.2\%) e infección de vías urinarias (5.2\%). Otras localizaciones como diarrea, flebitis y celulitis fueron menos frecuentes. Cabe destacar que se diagnosticaron 17 pacientes con conjuntivitis y que todos se encontraban en el mismo hospital, lo que pudo corresponder a un brote de conjuntivitis de probable etiología viral (cuadro II).

\section{Bacteriología de las IN}

Se colectó información microbiológica sobre un total de 66 hemocultivos relacionados con alguna IN; de 


\section{Cuadro I \\ Prevalencia de infecciones nosocomiales Y MORTALIDAD DE ACUERDO CON EL TIPO DE HOSPITAL. Ḿ́xico, 1996-1997}

Tipo de hospital

$\begin{array}{ccc}\text { Número } & \begin{array}{c}\text { Prevalencia } \\ \text { de IN } \\ \text { (IC95\%) }\end{array} & \begin{array}{c}\text { Tasa de mor- } \\ \text { talidad general } \\ \text { (IC95\%) }\end{array}\end{array}$

Unidades de pediatría

$\begin{array}{llll}\text { en hospitales generales } & 159 & 6.2(3.0-8.9) & 4.2(2.5-8.7)\end{array}$

\begin{tabular}{llll}
\hline Hospitales pediátricos de 20. nivel & 357 & $8.4(7.9-13.7)$ & $5.8(3.3-8.1)$ \\
\hline
\end{tabular}

Hospitales pediátricos de 3er. nivel $\quad 667 \quad 15.4$ (12.5-17.5) $\quad 6.3(4.6-7.9)$

\begin{tabular}{llll}
\hline Todos los hospitales & 1183 & $9.8(8.1-11.6)$ & $4.8(4.5-6.9)$
\end{tabular}

\section{Cuadro II \\ Sitios DE IN FECCIÓN \\ NOSOCOMIAL EN NiÑos HOSPITALIZA Dos. México, 1996-1997}

Sitio

Número (\%)

Neumonía

$29(25.4)$

Sepsis clínica

$16(13.8)$

Bacteriemia 6 (5.2)

Tracto urinario $6(5.2)$

Herida quirúrgica $5(4.3)$

Vías respiratorias altas $5(4.3)$

Peritonitis $4(3.4)$

Celulitis en sitio venopunción $4(3.4)$

Candidiasis sistémica $3(2.6)$

Diarrea $2(1.7)$

\begin{tabular}{ll}
\hline Conjuntivitis & $17(14.6)$ \\
\hline 0 tras & $19(16.4)$
\end{tabular}

éstos, 49 (74\%) fueron positivos. Los microrganismos identificados en estos hemocultivos fueron: Klebsiella pneumoniae (31\%), Escherichia coli (10\%), Pseudomonas spp. $(8.2 \%)$, S. aureus (8.2\%), Candida spp. (6.1\%) y $S$. coagulasa negativo (6.1\%).

\section{Factores de riesgo}

Para determinar los factores de riesgo asociados con IN en niños, se compararon las exposiciones previas al inicio de la infección en los infectados, con exposiciones el día de la encuesta en los infectados. El análisis bivariado se presenta en el cuadro III. Además de ser recién nacido prematuro o presentar desnutrición, se encontraron siete exposiciones (catéter intravenoso, nutrición parenteral, ventilación mecánica, catéter urinario, diálisis peritoneal, sonda nasogástrica y cirugía) significativamente asociadas $(p<0.05)$ con IN. Sin embargo, en el análisis de regresión logística para ajustar factores de confusión, únicamente cuatro variables permanecieron independientemente asociadas con una IN: exposición a catéteres intravenosos (RM 3.3, IC95\% 1.0-5.9), el uso de nutrición parenteral (RM 2.1, IC95\% 1.0-4.5), el uso de ventilación mecánica (RM 2.3, IC95\% 1.2-4.1) y ser recién nacido con peso menor a $2500 \mathrm{~g}$ (RM 2.6, IC95\% 1.0-6.8).

\section{Mortalidad asociada y atribuible}

En este estudio también se investigó la asociación de estas infecciones con la mortalidad en un total de 1056 niños, para lo cual se contó con información completa sobre sus condiciones al egreso. Hubo un total de 51 defunciones con una tasa de mortalidad general de $4.8 \%$; no obstante, hubo una importante variación en la mortalidad entre los hospitales participantes, pues osciló entre 2.2 y 9\%. Asimismo, se encontraron dife-

\section{Cuadro III \\ Características generales, procedimientos INVASIVOSY FACTORES DE RIESGO CRUDOS. MÉxıco, 1996-1997}

\begin{tabular}{|c|c|c|c|}
\hline \multirow[b]{2}{*}{ Características } & \multicolumn{3}{|c|}{ Porcentaje con la característica } \\
\hline & Infectados & No infectados & R. M. (IC95\%) \\
\hline \multicolumn{4}{|l|}{ Recién nacido } \\
\hline$<=2500 \mathrm{~g}$ & 22.7 & 9.2 & $2.9(1.0-8.6)$ \\
\hline$>2500 \mathrm{~g}$ & 77.2 & 90.8 & Referencia \\
\hline \multicolumn{4}{|l|}{0 tros grupos } \\
\hline$<1$ año & 54.4 & 49.2 & $1.3(0.9-2.0)$ \\
\hline $1-2$ años & 34.6 & 24.1 & $2.1(1.2-3.4)$ \\
\hline 2.5 años & 11.9 & 10.7 & $1.6(0.8-3.2)$ \\
\hline $5+$ años & 30.7 & 44.3 & Referencia \\
\hline Cáncer-neutropénico & 3.0 & 1.6 & $1.9(0.6-6.3)$ \\
\hline Desnutrido & 35.6 & 17.5 & $2.6(1.7-4.0)$ \\
\hline
\end{tabular}

Procedimientos invasivos

\begin{tabular}{lrrr} 
Soluciones IV & 53.5 & 56.9 & $0.9(0.6-1.3)$ \\
\hline Catéteres IV & 42.6 & 12.7 & $5.1(3.2-7.9)$ \\
\hline N utrición parenteral & 18.8 & 3.9 & $5.4(3.1-10)$ \\
\hline Ventilación mecánica & 31.7 & 9.5 & $4.4(2.8-7.2)$ \\
\hline Catéter urinario & 18.8 & 6.5 & $3.3(1.9-5.9)$ \\
\hline Diálisis peritoneal & 2.9 & 0.3 & $9.3(2.1-40)$ \\
\hline Traqueostomía & 0.0 & 0.7 & $0.0(0-5.8)$ \\
\hline Tubo nasogástrico & 36.0 & 13.8 & $3.5(2.2-5.5)$ \\
\hline Cirugía & 33.6 & 23.7 & $1.6(1.1-2.5)$ \\
\hline \multicolumn{1}{c}{ - Contaminada } & 2.0 & 0.3 & $6.8(1.3-34)$ \\
\hline
\end{tabular}

0 tras características

$\begin{array}{llll}\text { Mortalidad } & 10.5 & 4.9 & 2.9(1.1-6.8)\end{array}$


rencias en la mortalidad cruda de acuerdo con el grupo de edad y la presencia o no de una IN; la mortalidad en los menores de un año de edad fue significativamente mayor que en los de más de un año (8.3 vs $1.5 \%, p<0.01$ ), y en los pacientes con IN también fue más alta que en los que no las padecían (10.5 vs $4.9 \%, p<0.01)$. Según el sitio de infección, los pacientes con candidiasis tuvieron una mortalidad de $33 \%$; los que tenían sepsis, de $17 \%$, y los diagnosticados con neumonía, de $8 \%$.

En un modelo multivariado de IN se encontraron tres factores que predicen independientemente y en forma significativa el riesgo de morir por una IN: recién nacido (RM 3.1, IC95\% 1.8-5.5), desnutrición (RM 1.8, IC95\% 1.0-3.3) y sepsis nosocomial (RM 3.6, IC95\% 1.0-14.0). La variable de recién nacido se sustituyó por otra que agrupaba a todos los menores de un año, la cual a su vez también fue muy significativa (RM 5.7, IC95\% 2.7-11.8).

El riesgo de morir, ajustado por grupo de edad y desnutrición, fue más del doble en los pacientes con IN que en los referentes (RM 2.6, IC95\% 1.3-5.1). Además de determinar la magnitud de la asociación entre IN y mortalidad, también se consideró interesante estimar la proporción de muertes atribuibles a una IN en la población de pacientes pediátricos hospitalizados. La mortalidad atribuible a IN en estos hospitales fue de $15 \%$.

\section{Discusión}

Estos resultados permiten documentar que alrededor de uno de cada 10 niños hospitalizados adquiere una IN. De hecho, se puede inferir, con un intervalo de confianza de $95 \%$, que la prevalencia en pediatría está entre 8 y 11 IN por cada 100 niños hospitalizados. De particular importancia es el límite de confianza inferior, ya que es la medida que podrían usar los hospitales como referencia para disminuir sus tasas de infección. Si bien las tasas de infección para cada hospital podrían estar sujetas a variabilidad, el tamaño de muestra con más de 1000 observaciones hace muy estable al estimador global de prevalencia y proporciona un valor de referencia para estimar un rango de prevalencia de IN en pediatría de acuerdo con el tipo de hospital. Es importante observar cómo la tasa de IN aumenta de acuerdo con la complejidad del hospital. La tasa de prevalencia más alta se encontró en hospitales donde había pacientes con mayor severidad de enfermedad $y$, muy probablemente, sujetos con un mayor número de procedimientos invasivos, de tal manera que su riesgo de contraer una infección también aumentaba. Resultaría interesante comparar las tasas de infección entre hospitales, y para realizar comparaciones adecuadas sería necesario contar con tasas ajustadas y con un número mayor de observaciones en cada hospital. Estas tasas tendrían la ventaja de que, una vez habiendo ajustado por la gravedad de los pacientes, la complejidad del tratamiento, la comorbilidad, el tamaño y el tipo de hospital, toda diferencia residual podría atribuirse a diferencias en la calidad de la atención entre esos hospitales.

Este estudio representa uno de los intentos interinstitucionales más importantes hechos en México para caracterizar el problema de las IN en niños y proporciona información basal para evaluar intervenciones futuras.

Esta evaluación rápida, empleando una metodología y definiciones estándar, ha permitido identificar a la sepsis y a la neumonía nosocomial como las principales formas clínicas de IN, así como a K. pneumoniae como el principal microrganismo causante de las infecciones mencionadas en niños hospitalizados. Estos datos difieren de lo notificado en la literatura internacional, donde se indica que los estafilococos son los principales causantes de IN.$^{11}$ En México se identifica consistentemente a las enterobacterias como los agentes causales más importantes de IN en niños..$^{6-8}$

La prevención y el control de infecciones en hospitales requiere del conocimiento sobre incidencia, distribución, factores de riesgo, reservorios y propagación de agentes infecciosos entre la población de enfermos hospitalizados. Para hospitales con pocos recursos las actividades deben concentrarse en la prevención de entidades específicas (neumonías y sepsis), grupos de riesgo (recién nacidos), o servicios con alta frecuencia de procedimientos invasivos que manejan pacientes graves, como las unidades de terapia intensiva. La puesta en marcha de programas selectivos, podría llegar a ser más importante que los programas universales de vigilancia, que corren el riesgo de que las limitaciones de recursos diluyan el esfuerzo. ${ }^{17} \mathrm{La}$ efectividad de los programas de control depende de muchos factores, pero tal vez el más importante sea el de los recursos disponibles. En muchos hospitales mexicanos el equipo de control de infecciones no reúne los estándares recomendados para los hospitales estadunidenses. Es por ello que, dependiendo de los recursos humanos, la vigilancia de IN puede ser de dos tipos: a) vigilancia universal, la cual cubre todo el hospital en forma permanente, $y b$ ) vigilancia selectiva, que se efectúa cuando hay pocos recursos y se concentra en servicios prioritarios o en áreas de mayor riesgo. De acuerdo con los resultados de este estudio, algunos programas específicos tendrían prioridad. Por ejemplo, la identificación de factores de riesgo tie- 
ne implicaciones preventivas, y es de enorme utilidad para dirigir programas para el control de neumonías y sepsis/bacteriemia.

Si bien los programas universales como aquellos que promueven el lavado de manos son muy importantes, también se debe reconocer la importancia de programas específicos tales como la vigilancia, la supervisión y el cuidado de catéteres intravasculares, así como el cuidado especial para niños que están bajo ventilación mecánica. Así por ejemplo, cerca de la mitad de las IN corresponden a sepsis/bacteriemia y neumonías. Estas infecciones son relevantes no sólo por su frecuencia sino también por su elevada mortalidad. Debido a que están relacionadas con dos factores de riesgo (uso de catéteres intravasculares y de ventilación mecánica), desde el punto de vista epidemiológico su identificación debe ir seguida del diseño de programas preventivos. El énfasis radica no sólo en vigilar tasas de infecciones sino en monitorear los factores que las condicionan. Para ello, además de monitorear las medidas de efectos (tasas de infección), se deben vigilar los procesos de atención que dan lugar a esos efectos (manejo de catéteres y de ventilación mecánica). ${ }^{18}$ Por ejemplo, al estudiar las causas de bacteriemia en un hospital de Guanajuato, Macías y colaboradores ${ }^{19}$ demostraron que hasta $6.8 \%$ de las soluciones parenterales que se administraban a los niños estaban contaminadas principalmente con enterobacterias.

Para prevenir complicaciones y asegurar la calidad de los procesos de atención, es fundamental vigilar la instalación de catéteres, su permanencia y curación, y asegurar la esterilidad de la nutrición o de las soluciones parenterales que se administran. Una intervención que ha resultado eficaz en este sentido ha sido la creación de equipos de terapia intravenosa notificados por Volkow. ${ }^{20}$ Posiblemente otra intervención para reducir la frecuencia de bacteriemias es la creación de centros de mezclas en hospitales, lo cual garantizaría que toda la terapia intravenosa se preparara bajo estrictas condiciones de esterilidad. Por otro lado, el uso de asistencia ventilatoria como factor de riesgo para neumonía nosocomial obliga a garantizar la esterilidad del equipo de inhaloterapia, las bolsas de ventilación y la correcta aspiración de secreciones en pacientes con intubación endotraqueal, de tal manera que se eviten estas complicaciones infecciosas.

Por otra parte, resulta inquietante el hecho de que las IN consuman más recursos diagnósticos y terapéuticos para su manejo, pues incluso hay una buena proporción de esos pacientes con manejo intensivo. Así por ejemplo, en un estudio en el Hospital Pediátrico del Centro Médico Nacional del IMSS se encontró que los costos de terapia intensiva pediátrica sumaban
9297 pesos día/cama; sin embargo, el costo promedio global en los neonatos era muy superior que en los niños de mayor edad (\$ 110000.00 vs \$ 76000.00 ). ${ }^{21}$ Con estos antecedentes, se optó por hacer un ejercicio para estimar el impacto de estas infecciones en los costos de hospitalización, para lo cual se tomaron los días de estancia hospitalaria como una medida próxima al uso total de recursos hospitalarios. Para determinar el costo día/cama se realizó una encuesta en hospitales públicos que atienden niños. Se encontró que, después de ajustar por la hospitalización previa a la infección, los pacientes con IN tenían un exceso de cinco días de hospitalización. Al extrapolar estos datos al total de egresos anuales de niños en México, se detectó un exceso de 238500 días/cama al año por IN, que representarían un costo anual, para el sistema de salud, de 194 millones de pesos. ${ }^{22} \mathrm{Si}$ bien el incremento en costos es un argumento suficiente para desarrollar programas de control, la discapacidad y la mortalidad causadas por estas infecciones son todavía más preocupantes.

Además de mostrar que el riesgo de morir fue del doble en pacientes con IN, los resultados de este estudio también muestran que la proporción de muertes atribuibles a una IN en la población de pacientes pediátricos hospitalizados es de $15 \%$. El resto de las muertes podría ser consecuencia de la enfermedad de base, de otros diagnósticos y de complicaciones. Ese porcentaje también representa la proporción de muertes que podrían evitarse al prevenir esas infecciones, y sirve de base para estimar el impacto potencial de algunos programas preventivos. Así por ejemplo, si se extrapolan los datos de mortalidad a la población de 500000 niños que se hospitalizan anualmente, asumiendo una mortalidad de $5 \%$ y que de ellas $15 \%$ son atribuibles a IN, ésta equivale a 3750 muertes anuales en niños, mismas que podrían ser atribuibles a estas infecciones en México.

Este estudio también enseña algunas lecciones; este tipo de encuestas representan una alternativa económica y práctica para caracterizar la epidemiología de las IN y permiten tomar acciones de control sobre todo en hospitales que no cuentan con recursos para establecer programas permanentes de vigilancia universal. Es importante hacer énfasis en la consistencia de la información usando definiciones operacionales que reduzcan al máximo los errores por mala clasificación de caso, sobre todo cuando se pretende hacer comparaciones en estudios multicéntricos. Algunas revisiones sobre los métodos para mejorar la calidad de los servicios clínicos señalan que la colaboración interinstitucional es una estrategia para obtener avances importantes en la calidad de la atención. ${ }^{23}$ Por ejem- 
plo, la información sobre tasas de infección de estudios multicéntricos puede actuar como incentivo para reducir variabilidad entre instituciones y permite contar con una medida de referencia sobre la cual se marquen objetivos. La conformación de grupos de expertos entre instituciones, el establecimiento de criterios comunes para la definición de indicadores, el hecho de poder compartir experiencias exitosas, el diseño de programas preventivos basados en consenso, el desarrollo de metodologías de evaluación bajo supervisión de grupos externos a la institución y la definición de metas sujetas a verificación en tiempos establecidos, son algunos de los productos más importantes que estas colaboraciones multicéntricas pueden lograr y que difícilmente pueden obtenerse en los hospitales cuando operan en forma individual.

Es obvio que, debido a la naturaleza transversal del estudio, los resultados podrán tener algunas limitaciones. Primero, la tasa de prevalencia depende de la incidencia y de la duración de la enfermedad; por lo tanto, aquellas de mayor duración tienen también mayor probabilidad de ser detectadas, mientras que las de corta duración pueden ser subestimadas. Así, llama la atención la baja frecuencia de bacteriemias a pesar de que en otros informes éstas representan hasta $10 \%$ de las IN en niños. Segundo, la baja prevalencia de otras entidades clínicas, como la enfermedad diarreica, podría deberse a su variación estacional y a que no se captura en estudios de prevalencia lápsica.

En conclusión, los resultados de este trabajo permiten caracterizar la epidemiología de las IN en pediatría e ilustran el enorme potencial para prevenir estas infecciones, abatir costos y reducir la mortalidad, toda vez que se mejora la calidad de la atención médica. El impacto de estas infecciones, no sólo en costos, sino también en discapacidad y muerte, es el argumento más importante para desarrollar programas de garantía de calidad relacionados con el control de IN. La revisión de procesos como el cuidado de catéteres o el correcto manejo de pacientes con ventilación asistida es prioritaria y puede tener un gran impacto. Con frecuencia se argumenta la falta de recursos materiales en hospitales públicos; empero, esto es sólo parte del problema ya que hay una falta de mecanismos gerenciales para hacer un uso más eficiente de los escasos recursos disponibles. Se requiere revisar en forma crítica la cultura en los hospitales, romper la resistencia al cambio y organizar los recursos humanos creando equipos funcionales de trabajo y brindando adiestramiento en servicio y educación continua al personal. Finalmente, la información que proporciona este estudio sobre la prevalencia de IN en pediatría permitirá registrar los avances para su control.

\section{Referencias}

1. Haley RW, Culver DH, W hite JW, Morgan W M, Emori TG. The nationwide nosocomial infection rate:A new for vital statistics.Am J Epidemiol 1985;121;159-167.

2. Haley RW, Culver DH,W hite JW, Morgan W M, EmoriTG, MunnVP et al. The efficacy of infection surveillance and control programs in preventing nosocomial infections in US hospitals. Am J Epidemiol 1985;121:182-205. 3. Cruse PHE, Ford R. The epidemiology of wound infection: A 10-year prospective study of 62939 wounds. Surg C lin N orth A m 1980;60:27-40. 4. Avila-Figueroa R, Ramírez-G alván L, Alpuche-Aranda C, ArredondoGarcía JL, Santos-Preciado JI. Infecciones nosocomiales en un hospital pediátrico. Salud Publica Mex 1986;6:616-622.

5. León RA, Cashat CM, Avila-Figueroa FC, Aranda PE, Martínez G, Santos-Preciado Jl. Infecciones nosocomiales en el Hospital Infantil de México. Enf Infec Microbiol 1996;16(4):219-223.

6. G onzález SN , C oia LJJ, Saavedra BMA. Infecciones noso comiales: epidemiología del problema en el Instituto $\mathrm{N}$ acional de Pediatría. Rev Enfermedades Infecc Pediatr 1996;10:47-53.

7. Padilla BG, G uiscafré GH, Martínez GM, Vargas RR, Palacios TJ, Muñoz $H O$. Epidemiología de las infecciones nosocomiales en el hospital pediátrico. Salud Publica Mex 1986;28:599-610.

8. Avila-Figueroa C. Infecciones nosocomiales en recién nacidos. Bol Med Hosp Infant Mex 1988:46:86-89.

9. Mussaret ZJ, Ponce de León RS, Flores CJ, Monearla BD. Infecciones nosocomiales en una unidad de pediatría. Bol Med Hosp Infant Mex 1988:54:415-423.

10. Ibarra-C olado JE, Méndez-Hernández S, C ortés-C astillo LF. Infecciones hospitalarias en niños en un hospital general. Bol. Med Hosp Infant Mex 1991;11:820-825.

11. JarvisW R. Epidemiology of nosocomial infections in pediatric patients. Pediatr Infect D is J 1987;6:344-351.

12. Red Hospitalaria deVigilancia Epidemiológica (RHOVE). Coordinación de Vigilancia Epidemiológica. Subsecretaría de Prevención y Control de Enfermedades, Secretaría de Salud México, 1997.

13. Garner JS, Jarvis W R, Emori TG, Horan TC, Highes JM. CDC definitions for nosocomial infections. Am J Infect Control 1988;16:28-40.

14. D onowitz LG. Hospital acquired infection in the pediatric patient. Baltimore:W illiams and W ilkins, 1988.

15. Greeland S. Modeling and variable selection in epidemiologic analysis. Am J Public Health 1989;79:340-349.

16. Kelsey JK, Thompson W 0 , Evans AS. Methods in observational epidemiology. N ueva York: 0 xford University Press, 1986.

17.W idmer AF. Infection control and preventions strategies in the ICU. Intensive Care Med 1994;20:S7-S11.

18. Baker O G. Process surveillance: An epidemiologic challenge for health care organizations. Am J Infect Control 1977;25:96-101.

19. Macías-Hernández AE, H ernández-Ramos I, Muñoz-Barret JM, Juan M, Vargas SE, Guerrero MFJ et al. Pediatric primary gramnegative nosocomial bacteremia:A possible relationship with infusate contamination. Infect C ontrol Hosp Epidemiol 1996;17:276-280.

20. Volkow P, Sánchez-Mejorada G, Lazo DVS, Vázquez C, Téllez 0, Báez $\mathrm{RM}$ et al. Experience of an intravenous therapy team at the Instituto $\mathrm{Na}$ cional de Cardiología (Mexico) with a long-lasting, low-cost silastic venous catheter. Clin Infect D is 1994;18:719-725.

21. $N$ avarrete $S$, A rmengol G, Avila-Figueroa C. Evaluación económica de infecciones nosocomiales en unidades de cuidados intensivos pediátricos. Enferm Infecc Microbiol 1996;16:26.

22. Avila-Figueroa C. Evaluación del impacto económico y la mortalidad de las infecciones nosocomiales en pediatría. Enferm Infecc Microbiol 1996;16:53.

23. Plsek PE. Collaborating across organizational boundaries to improve the quality of care. Am J Infect Control 1997;25:85-95. 